\title{
Investigations of impact biomechanics for penetrating ballistic cases
}

\author{
A. Awoukeng-Goumtcha*, L. Taddei, F. Tostain and S. Roth \\ University of Technology of Belfort-Montbéliard, IRTES-M3M Laboratory, 90010 Belfort, France
}

\begin{abstract}
This study aims to investigate the penetration of a projectile into a surrogate human tissue numerically, using Finite Element (FE) simulation. 20\% Balistic Gelatin material (BG) is simulated with an elasto-plastic hydrodynamic constitutive law, and then impacted by steel spheres at different velocities. The results from the FE simulations are compared with existing experimental data and other analytical equations from the literature. To our knowledge, this is the first study to investigate a projectile penetration by numerical simulation, and then compare the results with analytical and experimental data from previous studies. This developed model gives encouraging results for further investigations of penetrating impact of projectile in the human body.
\end{abstract}

Keywords: Penetrating impact, finite element analysis, ballistic, biomechanics, human surrogate

\section{Introduction}

Since the number of trauma have largely increased in an impact biomechanics context, to understand the effects of blunt injuries is very important today [1]. The human body can be severely impacted by important mass with relative low velocity and very small mass with very high velocity (ballistic impact) [2]. Most studies related to ballistic or automotive impacts have been investigated experimentally. However, studies on multi-organ injuries, named "traumatic blast injuries" tend to use a combination of experiment and numerical modelling to assess the consequences of the human body [3-6]. Indeed, the development of computer science has made it possible to develop robust and realistic models to quantify local and global damages in traumatic blast injuries [3,4]. These models have been used to predict the severity of the hemorrhage, injury scale of various organs such as lung or the brain, and to simulate the kinematic of the human body under explosion loadings $[5,6]$.

Both numerical simulations and experimental tests are explored in the context of ballistic impacts: many experimental studies have been carried out to assess the ballistic impacts on the human body. In general, these experiments have been conducted on human tissue surrogate such as Ballistic Gelatin (BG), which behaves like human soft tissue [7-14]. These studies focus on the characterizations of material and bullet penetrating behaviors $[11,15,16]$, penetrating damages of the penetrated tissue, influence of the temperature and strain rates on tissue damage [8,10,11,16-18]. All these studies have

\footnotetext{
*Corresponding author: A. Awoukeng-Goumtcha, University of Technology of Belfort-Montbéliard, IRTES-M3M Laboratory, 90010 Belfort, France. Tel.: + 33 (0)3 845820 16; Fax: + 33 (0)3 845832 86; E-mail: aristide.awoukenggoumtcha@utbm.fr.
}

0959-2989/14/\$27.50 @ 2014 - IOS Press and the authors. 
included interesting results from numerical investigation (mechanical parameters for implementation of constitutive law) and validated the FE model (behavior of the projectile in the material and penetration time history). In this context, the present study investigates the behavior of $20 \%$ gelatin under high speed loading by numerical simulation and FE modelling. The results are compared to experimental data [12,19] and an analytical model [20-23] available in the literature. Different configurations of ballistic test were tested: the impact velocity varies from $240 \mathrm{~m} / \mathrm{s}$ to $2229 \mathrm{~m} / \mathrm{s}$; the projectile mass ranges from $0.055 \mathrm{~g}$ to $1.03 \mathrm{~g}$. Therefore, numerical simulations of penetrating impact into human surrogate are the first step in the modeling of ballistic penetration, and it could be implemented into a human biomechanical model for the investigation of wound ballistics.

\section{Methodology}

\subsection{Numerical model of ballistic impact into $B G$}

In order to simulate the penetrating ballistic impact and compare the numerical results to analytical solutions and experimental data, a 3D numerical model of the gelatin and steel sphere was generated. All numerical simulations were carried out using the finite element code Radioss (Altair@) [24]. This explicit code uses the central difference method as an integration scheme. The gelatin FE model is composed of $1 \mathrm{~mm}$ constant mesh in the impact zone with a linear bias density until the external surface in order to optimize the number of elements and the computational time. To avoid potential issues linked to bound effects on the external surface, we used the target cylinder with $252 \mathrm{~mm}$ in diameter, which is 40 times bigger than the maximum diameter of steel sphere $(6.3 \mathrm{~mm})$. Gelatin mesh is composed of 4340008 -nodes brick elements (Figure 1). The mesh size in the center of the sample is $0.5 \mathrm{~mm}$, which is comparable to the smallest diameter of the projectile.

The material properties were implemented in the model following an elasto-plastic hydrodynamic material law. Indeed, it has been shown that during ballistic impact, the elastic and plastic regions of soft tissues, such as gelatin, are stimulated following a classic law of Johnson-Cook - - with a small plastic contribution [25]. Then the hydrodynamic part is effective at high velocity when the pressure behavior function of the volume is non-linear [13,17]. This contribution is simulated with the polynomial form Eq. (4). A Johnson-Cook law is used to model the stress-strain relationship under tension (Eq. (1)) with A, B, $\mathrm{n}$ are the yield stress, the hardening modulus and the hardening exponent respectively.

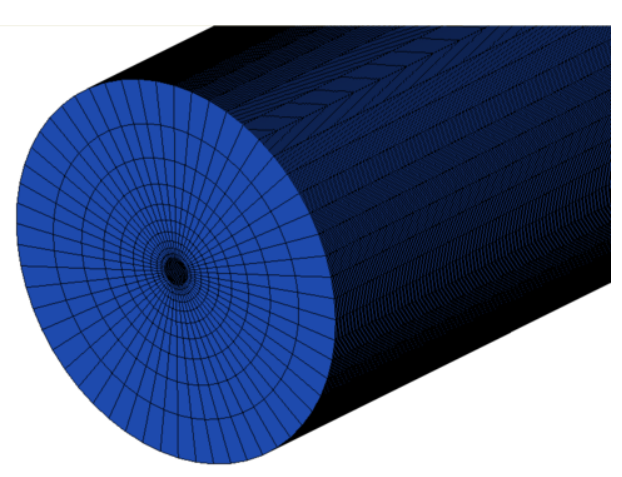

Fig. 1. 3D finite element mesh of gelatin. 


$$
\sigma_{\mathrm{p}}=\mathrm{A}+\mathrm{B} \varepsilon^{\mathrm{n}}
$$

As the material is considered as fragile, the plastic behavior has a negligible effect. The compression behavior is given by a polynomial expression Eq. (4), based on the Mie-Grüneisen EOS $[17,26]$ which consider the pressure evolution as a function of the volume and the temperature (Eq. (2)). pst is the isothermal pressure function of the volume $\mathrm{V}$ at a temperature $\mathrm{T}_{0}$ and $\Delta \mathrm{pth}$ is the gradient of pressure due to the temperature variation.

$$
\mathrm{p}(\mathrm{V}, \mathrm{T})=\mathrm{p}_{\mathrm{st}}\left(\mathrm{V}, \mathrm{T}_{0}\right)+\Delta \mathrm{p}_{\mathrm{th}}(\mathrm{V}, \mathrm{T})
$$

According to experiments in high compression of the BG [13,17], the shock state (defined by the relation between the shock velocity $u_{s}$ and the particle or material velocity $u_{p}$ ) can be described by the shock Hugoniot function Eq. (3) where the behavior depend of two constants which are the sound speed $\mathrm{c}$ and the linear coefficient of Hugoniot $\mathrm{s}$. Then, the pressure behavior could be described by four material properties which are the density $\rho$, the sound speed in the material $c$, the Grüneisen constant $\Gamma_{0}$ and the Hugoniot coefficient s.

$$
\begin{aligned}
& \text { us }=\mathrm{c}+\mathrm{s} \text { up } \\
& P=C_{0}+C_{1} \mu+C_{2} \mu^{2}+C_{3} \mu^{3}+\left(C_{4}+C_{5} \mu\right) E_{v 0}
\end{aligned}
$$

with $\mu=1-\frac{\rho}{\rho_{0}}, \rho_{0}$, and $E_{v 0}$ are respectively the initial density and the energy per unit of initial volume. In the case of a condense environment (fluid or solid), the hydrodynamic constants $\mathrm{C}_{\mathrm{i}}$ are described by $[24,27]$ :

$$
\begin{aligned}
& C_{0}=P_{0} \quad C_{1}=\rho_{0} c_{0}^{2}-\frac{\Gamma_{0}}{2} P_{0} \quad C_{2}=\rho_{0} c_{0}^{2}(2 s-1)-\frac{\Gamma_{0}}{2} \rho_{0} c_{0}^{2} \\
& C_{3}=\rho_{0} c_{0}^{2}(2 s-1)(3 s-1)-\frac{\Gamma_{0}}{2} \rho_{0} c_{0}^{2}(2 s-1) \quad C_{4}=C_{5}=\Gamma_{0}
\end{aligned}
$$

The modeling of gelatin ballistic is a complex task due the complexity of the material, especially at high strain rate. The literature concerning the material characterization of such material does not lead to consensus, and studies provide different mechanical parameters $[7,16,18]$.

Table 1

Mechanical parameters implemented in the elastic-plastic-hydrodynamic law

\begin{tabular}{lllllll}
\hline $\mathrm{E}(\mathrm{MPa})$ & $\mathrm{N}$ & $\mathrm{A}(\mathrm{MPa})$ & $\rho_{0}\left(\mathrm{~g} / \mathrm{cm}^{3}\right)$ & $\mathrm{C}_{0}(\mathrm{~m} / \mathrm{s})$ & $\mathrm{S}$ & $\Gamma_{0}$ \\
\hline 2.5 & 0.499 & 2 & 1.06 & 1520 & 1.87 & 0.17 \\
\hline
\end{tabular}


However, for this study, in order to take into account the elastic-plastic-hydrodynamic behavior, a set of mechanical parameters listed in Table 1 have been used and implemented in the FE code.

As a penetrating can lead to very high deformations of elements in the mesh at a numerical level, it is necessary to implement a failure model. Element deformation state had been used for element deletion when the deformation is higher than a threshold (0.8), which corresponds to the occurrence of cracks in gelatin specimens during compressive tests [7]. In order to compute correctly the interaction between gelatin and projectile, a classical interface was used between the two bodies involving computation of forces between master surface and slave nodes. The interaction between gelatin and steel sphere leads to high deformation of the gelatin, whereas no deformation of the projectile is observed due to the huge difference in terms of elasticity. In order to optimize the calculation time, the projectile were considered as rigid. The time pitch of the simulations depends on the mesh grid, the material laws, and the interface between the GB and the projectile. Finally for the impact configurations, the order of magnitude of the time step was $4.10^{-5} \mathrm{~ms}$, which was considered correct.

\subsection{Analytical model}

Experiment data are extracted from the study of Sturdivan (1978). In this research steel spheres impact $20 \%$ gelatin. The author presented penetration time history for different diameters $\mathrm{d}$ and striking velocities $V_{S}$ of steel sphere. Based on the Sturdivan's data and the Poncelet's equation, some analytical models were developed with rigid projectile [12,20,23]. The following Eq. (7) is the solution of Poncelet's equation Eq. (6) who determined empirically the equation that describes the acceleration of a projectile while penetrating through a material, where penetration constant $\mathrm{B}$ and $\mathrm{C}$ are functions of both projectile mass and material properties [12].

$$
-M \dot{V}=B V^{2}+C
$$

Given a striking velocity $\mathrm{V}_{0}$, the solution yields the penetration depth as a function of the current velocity:

$$
x(V)=\frac{M}{2 B} \ln \left(\frac{C+B V_{s}^{2}}{C+B V^{2}}\right)
$$

This Eq. (7) gives the penetration depth x (Eq. (8)), and velocity V (Eq. (9)), as a function of time:

$$
\begin{aligned}
& x(t)=\frac{M}{B}\left\{\ln \cos \left[\frac{\sqrt{B C}}{M}(t f-t)\right]-\ln \cos \left[\frac{\sqrt{B C}}{M} t f\right]\right\} \\
& V(t)=\sqrt{\frac{C}{B}} \tan \left[\frac{\sqrt{B C}}{M}(t f-t)\right]
\end{aligned}
$$

With $t_{f}$ the impact duration and $M$ given by:

$$
\mathrm{tf}=\frac{\mathrm{M}}{\sqrt{B C}} \tan ^{-1}\left(V_{S} \sqrt{\frac{B}{C}}\right) \text { With } \mathrm{B}=-\frac{\mathrm{C}_{\mathrm{d}} \rho_{\mathrm{t}} \pi \mathrm{d}^{2}}{8} \mathrm{C}=-\frac{\sigma_{\mathrm{t}} \pi \mathrm{d}^{2}}{4} \text { and } \mathrm{M}=-\frac{\pi \mathrm{d}^{3}}{6} \cdot \rho_{S}
$$


$\mathrm{V}_{0}$ : Impact Velocity

M: Mass of sphere

$\sigma_{\mathrm{t}}:$ Apparent target strengt (Wijk, $\left.2001[12]\right)$

$\rho_{\mathrm{s}}$ : Sphere density

$\rho_{\mathrm{t}}$ : Target density

$\mathrm{C}_{\mathrm{d}}$ : Drag coefficient

d: Diameter of sphere

$\mathrm{B}$ and $\mathrm{C}$ are determined from the study of Wijk et al. and are linked to the impact configurations.

In this study, a comparison between analytical model [12], experimental tests [19] (Sturdivan data), and the proposed FE model were conducted. The measure of penetration time history and velocity versus penetration curves were provided.

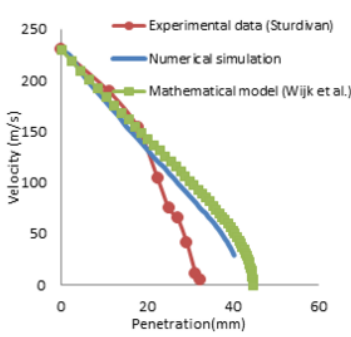

Gelatin is impacted by a $0.055 \mathrm{~g}$ projectile at

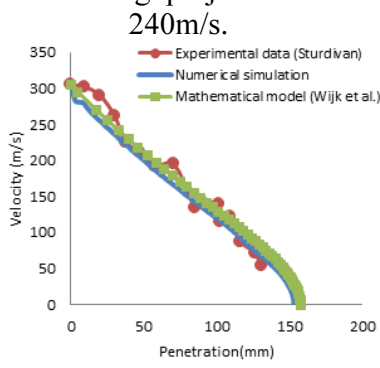

Gelatin is impacted by a

$1.03 \mathrm{~g}$ projectile at $305 \mathrm{~m} / \mathrm{s}$.

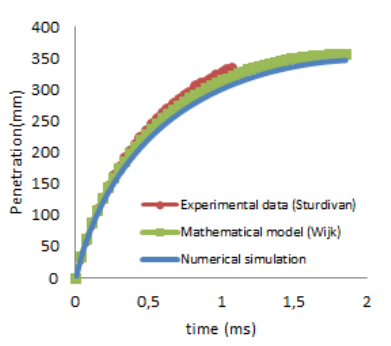

Gelatin is impacted by a $1.03 \mathrm{~g}$ projectile at $1022 \mathrm{~m} / \mathrm{s}$

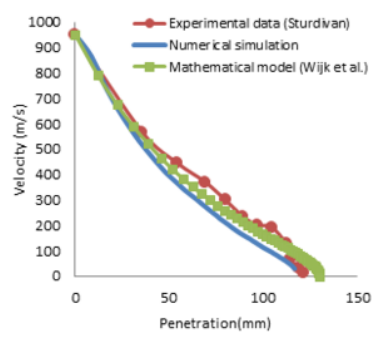

Gelatin is impacted by a $0.055 \mathrm{~g}$ projectile at $951 \mathrm{~m} / \mathrm{s}$.

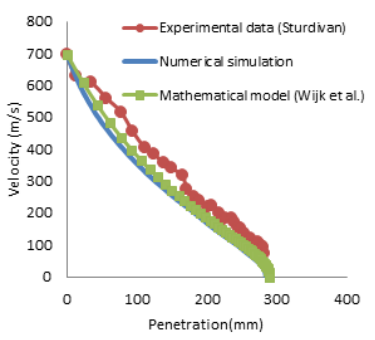

Gelatin is impacted by a $1.03 \mathrm{~g}$ projectile at $696 \mathrm{~m} / \mathrm{s}$.

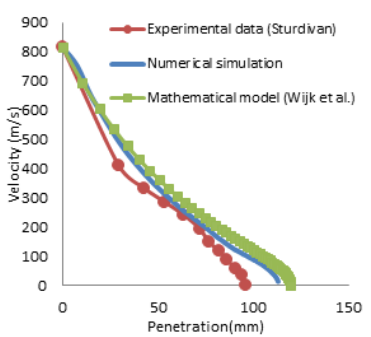

Gelatin is impacted by a $0.055 \mathrm{~g}$ projectile at $814 \mathrm{~m} / \mathrm{s}$.

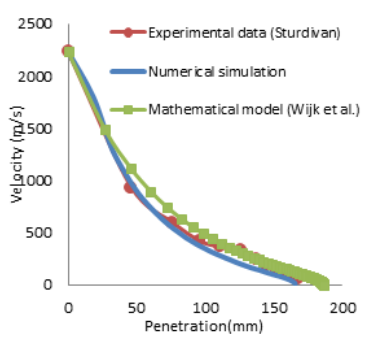

Gelatin is impacted by a $0.055 \mathrm{~g}$ projectile at

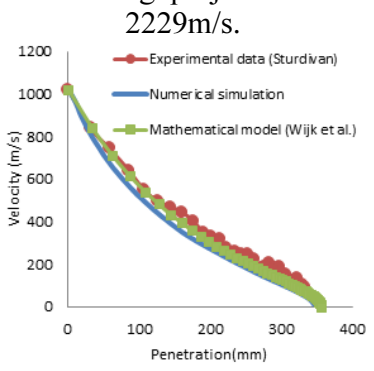

Gelatin is impacted by a $1.03 \mathrm{~g}$ projectile at $1022 \mathrm{~m} / \mathrm{s}$.

Fig. 2. Velocity versus penetration curves.
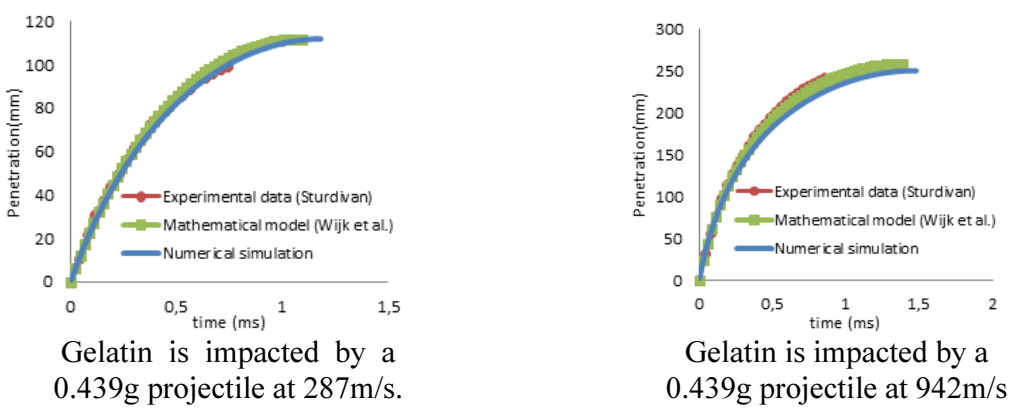

Gelatin is impacted by a $0.439 \mathrm{~g}$ projectile at $942 \mathrm{~m} / \mathrm{s}$

Fig. 3. Penetration history time. 


\section{Results}

Different figures provide numerical results compared to experimental data performed by Sturdivan (1978) and mathematical model developed by Wijk et al. (2001). Velocities versus penetration curves are Figure 2) as well as penetration time history (Figure 3) are reported. Based on Sturdivan's data, different diameters $\mathrm{d}$ and striking velocities Vs of steel sphere were simulated. For each impact configuration, the model reproduces reasonably the experimental and analytical response, as shown by Sturdivan et al [19].

It is important to notice that in addition to velocity versus penetration curves and penetration time history, the final penetration of the bullet (when the projectile stops in the material, at the end of the simulation), was also observed (Figure 3).

\section{Discussion, future work and conclusion}

\subsection{Discussion}

The numerical modeling of steel spheres impacting $20 \%$ gelatin is a key point in the understanding of the response of a ballistic penetrating impact. Indeed, BG has been frequently used as a tissue simulant in a variety of research scenarios appeared to be a reliable human tissue surrogate $[13,14,28]$. However, it has to be noticed that lots of biological components cannot be simulate with this kind of tissue surrogate, including blood, nerve or vessel. Hence, experimental and numerical approaches of ballistic impact using gelatin are the first step in studying high speed penetrating impact.

At the numerical level, the curves obtained with the present simulations give globally quite acceptable results, their responses being close to the experimental data [19] and the mathematical model [12].

The penetration error between numerical and experimental data, and between numerical and theoretical model is about $10 \%$ and $7 \%$ respectively (an average of ten cases). However, this difference is much higher when the gelatin is impacted by a $0.055 \mathrm{~g}$ projectile at $240 \mathrm{~m} / \mathrm{s}$. In this specific scenario, the final penetration from both numerical modeling and analytical model are slightly higher than described in Sturdivan's study. Here are several possible explanations for this discrepancy:

At analytical level, uncertainties remain on the drag coefficients as the gelatin is assumed to be similar to a Newtonian fluid. Moreover, the influence of hydrodynamics may decrease with the kinetic energy if the hydrodynamic factor is considered in the constitutive law. Then the final penetration is more likely to be influenced by mechanical factors. Based on this, the gelatin should be more considered as a soft structure than a fluid.

From a modeling point of view, the response could also be affected by numerical errors: although the energy balance of the simulations were checked (Hourglass Energy was considerably lower than the total Energy of the system due to anti-Hourglass formulation), errors linked to elements distortion still exist. This issue could be solved by the use of meshless numerical methods (e.g. Smooth Particle Hydrodynamics), which have been developed specially for large deformation to study penetrating impact [29]. Errors can also be explained by the influence of the temperature in experimental data. Material properties of BG are very sensitive to the temperature [12,17], and this parameter is not taken into account in the FE analysis or in the analytical model).Finally, materials are considered as homogeneous in numerical simulation. In reality, the mechanical properties and the penetration of the sphere will be altered when additional foreign bodies are added inside, such as bullets of water or air, [23]. Furthermore, non-ideal surface contact between gelatin and steel sphere has a great influence in 
the penetration properties. This needs further investigation for the development of numerical models [30].

\subsection{Future work}

Ballistic phenomena are complicated and involve several aspects of physics. Rifles and handguns have rifled barrels which give a rotational spin to the bullet along its longitudinal axis [14]. Therefore, the bullet projectile has two velocity components on the penetration axis: rotational and longitudinal. Both velocities should be taken into account for realistic ballistic impact at aerodynamic level. In terms of bullet material, the projectile has a damage response [31] and its trajectory is influenced by the bullet material degradation. Further studies will work on the simulations where rotational and longitudinal velocities of real bullet geometry are taken into consideration [30], and where bullets with realistic deformable material properties are applied. The objective of ballistic tests in BG is to reproduce penetrating impact in the human body. Even if the BG tends to reproduce the mechanical behavior of soft tissue, multi-layer numerical tests should be performed in order to mimic the different layers of tissue in the human body: skin, fat, muscle, water, organs, etc. This multi-layered model for "hard" material has already been studied in the literature [22]. Actual analytical (Wijk, et al. and Segletes, et al) and the present FE model has allowed us to determine the penetration and the velocity for one single layer: future studies will focus on studying the penetrating impact for multiple layers of soft tissue. Finally, the numerical formulation should be further improved in order to avoid a significant distortion of the mesh, especially for studies using meshless methods. At a numerical level, some improvements can also be done concerning the use of different schemes, for an improvement of the computation time $[32,33]$.

\subsection{Conclusion}

Normal penetrating impacts of rigid sphere on $20 \%$ Gelatin have been studied and a FE model was developed and compared to experimental data and analytical model given in the literature. Spheres of three diameters $(2.38,4.76,6.3 \mathrm{~mm})$ were used in the numerical model and the impact velocity ranged from 230 to $2229 \mathrm{~m} / \mathrm{s}$. The model reasonably reproduces experimental tests and the mathematical results as reported in literatures. Further, the numerical model can predict penetrating impact in BG in a realistic manner.

This study is a first step in the numerical investigation of high speed penetrating impact into a realistic human tissue surrogate, and could be implemented in a biomechanical model for an improvement of its biofidelity. The developed model can assist the further investigation of penetrating impact in the human body, with specific studies concerning protective device (body armor) and also at a medical level for investigations of wound ballistic and organs injuries.

\section{Acknowledgement}

Authors would like to thank the Region Franche-Comté, France, for their financial support. 


\section{References}

[1] D.C. Viano, Ian V. Lau, C. Asbury, A.I. King and P. Begeman, Biomechanics of the human chest, abdomen, and pelvis in lateral impact, Accident Analysis \& Prevention 21 (1989), 553-574.

[2] C. Bir, D. Viano and A. King, Development of biomechanical response corridors of the thorax to blunt ballistic impacts, Journal of Biomechanics 37 (2004), 73-79.

[3] R. Kannan and A.J. Przekwas, A near-infrared spectroscopy computational model for cerebral hemodynamics, International Journal for Numerical Methods in Biomedical Engineering 28 (2012), 1093-1106.

[4] R. Kannan and A. Przekwas, A computational model to detect and quantify a primary blast lung injury using nearinfrared optical tomography, International Journal for Numerical Methods in Biomedical Engineering 27 (2011), 13-28.

[5] X.G. Tan, R. Kannan and A.J. Przekwas, A comparative study of the human body finite element model under blast loadings, ASME 2012 International Mechanical Engineering Congress and Exposition, Biomedical and Biotechnology 2 (2012), 829-836.

[6] X.G. Tan, R. Kannan, A.J. Przekwas, K. Ott, T. Harrigan, J. Roberts and A. Merkle, An enhanced articulated human body model under c4 blast loadings, ASME 2012 International Mechanical Engineering Congress and Exposition, Biomedical and Biotechnology 2 (2012), 821-828.

[7] P. Moy, C.A. Gunnarsson and T. Weerasooriya, Tensile deformation and fracture of ballistic gelatin as a function of loading rate, SEM Annual Conference, Albuquerque New Mexico USA, Society for Experimental Mechanics Inc., 2009.

[8] C.P. Salisbury and D.S. Cronin, Mechanical properties of ballistic gelatin at high deformation rates, Experimental Mechanics 49 (2009), 829-840.

[9] F. Bresson, J. Ducouret, J. Peyré, C. Maréchal, R. Delille, T. Colard and X. Demondion, Experimental study of the expansion dynamic of $9 \mathrm{~mm}$ parabellum hollow point projectiles in ballistic gelatin, Forensic Science International 219 (2012), 113-118.

[10] D.S. Cronin and C. Falzon, Dynamic characterization and simulation of ballistic gelatin, SEM Annual Conference Albuquerque New Mexico USA, Society for Experimental Mechanics Inc., 2009.

[11] T.F. Juliano, P. Moy, A.M. Forster, T. Weerasooriya and M.R. VanLandingham, Multiscale mechanical characterization of biomimetic gels for army applications, Technical report, U.S. Army Research Laboratory, Weapons \& Materials Research Directorate, Aberdeen Proving Ground, MD, 21005, 2006.

[12] G. Wijk, A. Collin and R. Amiree, Sphere penetration into gelatine and board, 19th International Symposium of Ballistics 2 (2001), 1019-1027.

[13] K. Nagayama, Y. Mori, Y. Motegi and M. Nakahara, Shock hugoniot for biological materials, Shock Waves 15 (2006), $267-275$.

[14] V.J.M.D. Maio, Gunshot Wounds: Practical Aspects of Firearms, Ballistics, and Forensic Techniques, 2nd edi., CRC Press, USA, 1998.

[15] O. Mauzac, C. Paquier, E. Debord, F. Barbillon, P. Mabire and J.F. Jacquet, A substitute of gelatin for the measurement of dynamic back face deformation, Personal Armour System Symposium, 2010.

[16] D.S. Cronin and C. Falzon, Characterization of $10 \%$ ballistic gelatin to evaluate temperature, aging and strain rate effects, Experimental Mechanics 51 (2011), 1197-1206.

[17] M. Aihaiti and R.J. Hemley, Equation of state of ballistic gelatin (I) final report, Carnegie Institution of Washington, 5252 Broad Branch Rd. Washington, DC 20015, 2008.

[18] J. Kwon and G. Subhash, Compressive strain rate sensitivity of ballistic gelatin, Journal of Biomechanics 43 (2010), 420-425.

[19] L.M. Sturdivan, A mathematical model of penetration of chunky projectiles in a gelatin tissue simulant, Technical Report ARCSL-TR-78055, Chemical Systems Laboratory, Aberdeen Proving Ground, Maryland 21010, 1978.

[20] L. Liu, Y. Fan, W. Li and H. Liu, Cavity dynamics and drag force of high-speed penetration of rigid spheres into 10 wt\% gelatin, International Journal of Impact Engineering 50 (2012), 68-75.

[21] M.J. Forrestal and D.Y. Tzou, A spherical cavity-expansion penetration model for concrete targets, International Journal of Solids and Structures 34 (1997), 4127-4146.

[22] G.W. Stone, Projectile penetration into representative targets, Technical Report DE-AC04-94AL85000, Sandia National Laboratories, Albuquerque, New Mexico 87185 and Livermore, California 94550, 1994.

[23] S.B. Segletes, Modeling the penetration behavior of rigid spheres into ballistic gelatin, Final ARL-TR-4393, U.S. Army Research Laboratory, Aberdeen Proving Ground, MD 21005-5069, 2008.

[24] Altair Engineering, Radioss Theory Manual 11.0 version-Large Displacement Finite Element Analysis Chapter 9Material Laws, 2010

[25] J.S. Wilbeck, Impact behaviour of low strength projectiles, Technical Report AFML-TR-77-134, Air Force Materials Laboratory, 1978. 
[26] I. Chaieb, Analysis and simulation of residual stresses due to shot-blasting and laser shock prestressing mechanical treatments, Ph.D Dissertation, University of Reims Champagne-Ardenne, 2004.

[27] J-P. Cuq-Lelandais, M. Boustie and T. De Ressguier, Using hyperworks v 9.0 suite for high speed modelling with Radioss code (2009-2010).

[28] N.C. Nicholas and J.R. Welsch, Ballistic gelatin, Report, Institute for Non-Lethal Defense Technologies, The Pennsylvania State University, 2004.

[29] G. Portemont, Contribution to the numerical method development of hard and soft components interaction-Application to crash and impact context, Ph.D Dissertation, University of Valenciennes Hainaut Cambrésis, 2004.

[30] W. Goldsmith, Non-ideal projectile impact on targets, International Journal of Impact Engineering 22 (1999), 95-395.

[31] D. Datoc, Finite element analysis and modeling of a .38 lead round nose ballistic gelatin test, Ph.D Dissertation, California Polytechnic State University, 2010.

[32] C. Liang, R. Kannan and Z.J. Wang, A p-multigrid spectral difference method with explicit and implicit smoothers on unstructured triangular grids, Computers and fluids 38 (2009), 254-265.

[33] R. Kannan and Z.J. Wang, A study of viscous flux formulations for a p-multigrid, spectral volume Navier stokes solver, Journal of Scientific Computing 41 (2009), 165-199. 